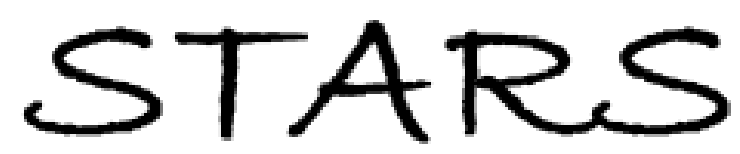

University of Central Florida

STARS

Faculty Bibliography 2000s

Faculty Bibliography

$1-1-2007$

\title{
Wireless pressure sensor using laser targeting of silicon carbide
}

Nabeel A. Riza

University of Central Florida

Farzan Ghauri

University of Central Florida

Frank Perez

Find similar works at: https://stars.library.ucf.edu/facultybib2000

University of Central Florida Libraries http://library.ucf.edu

This Article; Proceedings Paper is brought to you for free and open access by the Faculty Bibliography at STARS. It has been accepted for inclusion in Faculty Bibliography 2000 s by an authorized administrator of STARS. For more information, please contact STARS@ucf.edu.

\section{Recommended Citation}

Riza, Nabeel A.; Ghauri, Farzan; and Perez, Frank, "Wireless pressure sensor using laser targeting of silicon carbide" (2007). Faculty Bibliography 2000s. 7574.

https://stars.library.ucf.edu/facultybib2000/7574

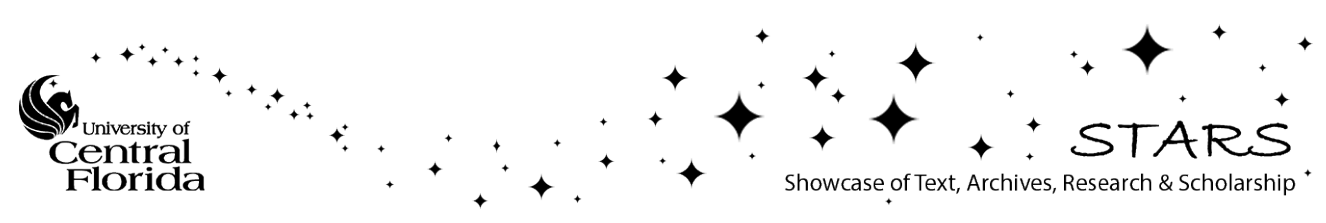




\section{Wireless pressure sensor using laser targeting of silicon carbide}

\author{
Nabeel A. Riza \\ Nuonics, Inc. \\ 1025 South Semoran Boulevard, Suite 1093 \\ Winter Park, Florida 32792 \\ and \\ University of Central Florida \\ College of Optics-CREOL \\ 4000 Central Florida Boulevard \\ Orlando, Florida 32816-2700
}

\author{
Farzan Ghauri \\ University of Central Florida \\ College of Optics-CREOL \\ 4000 Central Florida Boulevard \\ Orlando, Florida 32816-2700
}

\section{Frank Perez}

Nuonics, Inc.

1025 South Semoran Boulevard, Suite 1093

Winter Park, Florida 32792

\begin{abstract}
To the best of our knowledge, proposed is the first extremeenvironment wireless pressure sensor design using a remoted singlecrystal SiC chip within a pressurized capsule. A detailed theoretical analysis of the sensor system is performed, including the SiC chip's mechanical response within the pressure capsule and the pressure measurement technique's optical response based on image demagnification. The remote sensor was experimentally tested at room temperature for pressures up to $41 \mathrm{~atm}$, and the sensor response is consistent with the theoretical analysis. The demonstrated sensor has a current experimental resolution of 1.17 atm with a designed maximum pressure range of $140 \mathrm{~atm}$. Improved sensing resolution and range can be achieved via optimal selection of the SiC chip's dimensions and its seating in the pressure capsule. Applications for this sensor include extreme environments involving hot gases and corrosive fluids, as in power generation systems, oil field operations, and aerospace systems. () 2007 Society of Photo-Optical Instrumentation Engineers. [DOI: 10.1117/1.2432885]
\end{abstract}

Subject terms: optical sensors; pressure sensor; extreme environments.

Paper 060241 received Mar. 31, 2006; accepted for publication Jun. 26, 2006; published online Feb. 6, 2007. This paper is a revision of a paper presented at the SPIE conference on Optical Sensing II, Strasbourg, France, Apr. 2006. The paper presented there appears (unrefereed) in SPIE proceedings Vol. 6189.

\section{Introduction}

Today, fiber-optic sensors are used in industry for various sensing applications in aircraft, locomotives, bridges, and power systems. ${ }^{1}$ A particular industrial scenario is fossilfuel-based power generation systems, which fall into the category of extreme environments in terms of temperature, pressure, and caustic fluids and gases. Generation of clean energy is an important global concern. ${ }^{2}$ Early fossil-fuel power generation system designs show improved conversion efficiencies and lower greenhouse gas emissions when operating at extreme temperatures $\left(>1500^{\circ} \mathrm{C}\right)$ and pressures $(>50 \mathrm{~atm}){ }^{3}{ }^{3}$ These next-generation power production systems need long-lifetime high-reliability sensors for extreme temperature and pressure measurements. Most importantly, these sensors need to survive the extreme hightemperature, high-pressure chemical conditions, including corrosive fluids, hot gases, molten metals, and sludge/ waste. Of particular importance are extreme-environment pressure sensors, the subject of the present paper.

Pressure sensors have been built by utilizing the variation in the resistance or capacitance of a device under pressure. Prototype silicon carbide (SiC) high-temperature piezoresistive pressure sensors were batch-fabricated at the NASA John Glenn Research Center by producing the diaphragms using a chemical micromachining process, and the sensors showed promise and were demonstrated to operate up to $500^{\circ} \mathrm{C}^{4}$ Okojie et al. ${ }^{5}$ fabricated and tested piezoresistive pressure sensors at $1000 \mathrm{psi}(68 \mathrm{~atm})$ with full-scale output 40.66 and $20.03 \mathrm{mV}$ at 23 and $500^{\circ} \mathrm{C}$, respectively.

0091-3286/2007/\$25.00 @ 2007 SPIE
Ziermann et al. ${ }^{6}$ used $\mathrm{SiC}$ on insulator (SiCOI) to create a piezoresistive pressure sensor and tested its operation between room temperature and $500^{\circ} \mathrm{C}$. They reported the sensitivity of the device to be $20.2 \mu \mathrm{V} /(\mathrm{V} \mathrm{kPa})$ at room temperature. Since these $\mathrm{SiC}$ electronic sensors are based on the principle of piezoresistance, micropipe defects in $\mathrm{SiC}$ negatively impact performance when compared to silicon-based electronic sensors. Attempts have also been made to make $\mathrm{SiC}$ membrane optical microelectromechanical system (MEMS) fiber optic pressure sensors using sapphire fibers. ${ }^{7}$ Here, performance is shown for relatively low pressures (e.g., $20 \mathrm{psi}$ ) using thin $\mathrm{SiC}$ membranes. Moreover, all these SiC MEMS pressure sensors are wired nonpassive devices. In other words, electronic power and processing is done on the front-end chip or via a remoted fiber wire (e.g., sapphire fiber, which is limited by multimodal optical noise) that is simultaneously exposed to the changing extreme environment. In effect, all the processing in the chip (plus the fiber connection for the optical MEMS case) must withstand any extreme environmental effects. Thus the packaging of the remoting wire for the sensor becomes a key concern and limitation. Hence, works were implemented to produce a wireless electronic pressure sensor. ${ }^{8-10}$ These highlighted sensors require on-chip power plus electronics and contacts, which are nonrobust to high temperatures. Another design proposed ${ }^{11}$ is passive, nevertheless, it presently has limitations in the temperature $\left(<400^{\circ} \mathrm{C}\right)$ and pressure $(<7$ bars $)$ ranges of operation. In silicon technology, $p$ - $n$ junction-isolated piezoresistors are used as pressure sensors for temperatures less than $175^{\circ} \mathrm{C}$, and silicon-on-insulator (SOI) sensors for temperatures up to $500^{\circ} \mathrm{C}$. 
Other techniques have also been investigated to measure pressure. Leading fiber-optic sensors such as those using fiber Fabry-Perot interference or in-fiber Bragg gratings with wavelength-based processing also use the fiber wire for light delivery and light return, hence inherently possessing the limitations of wired devices for extreme environments. ${ }^{12-19}$ Optically reflective ${ }^{20}$ and interferometric ${ }^{21-24}$ techniques have also been investigated. The interferometric techniques were based on Fabry-Perot interferometer/cavity formed by etching a glass substrate or the tip of an optical fiber and enclosing the etched volume with a silicon diaphragm. The materials in these optical devices were glass and silicon, which will melt at the high temperature environment in fossil-fuel applications. Recently further work on optical pressure sensors has been reported, but all have their limitations due to the exposure of their nonrobust sensing element and/or their connecting fibers in the extreme power generation systems environment. These are listed in Refs. 25-32 All these fiber-based optical pressure sensors are wired designs.

Recently, a hybrid fiber-free-space optics approach to sensing was put forth that exploits both the fiber-based remoting capability and the minimally invasive nature of laser-targeted light beams incident on specific sensing front-end chips. ${ }^{33-35}$ Specifically for harsh environments, the use of single-crystal $6 \mathrm{H} \mathrm{SiC}$ has been suggested to enable temperature, pressure, and gas species sensing. It is for its material robustness to chemical attack, mechanical stability, and excellent thermal and optical properties that single-crystal $\mathrm{SiC}$ is chosen as the base optical sensor material. There are numerous polymer types of $\mathrm{SiC}$ such as $3 \mathrm{C}$ (cubic), 2H (hexagonal), 4H (hexagonal), 6H (hexagonal), and $15 \mathrm{R}$ (rhombohedral). The key point to note is the high $\left(2500^{\circ} \mathrm{C}\right)$ melting temperature of $6 \mathrm{H} \mathrm{SiC}$, which makes it ideal for extreme temperature conditions. In addition, its Young's elastic modulus, yield strength, and Poisson ratio prove it to be a mechanically robust material. These material properties make single-crystal high thickness (e.g., $300 \mu \mathrm{m}) 6 \mathrm{H} \mathrm{SiC}$ an ideal, reliable candidate for use in extreme environment sensors such as are needed for coalfired power plants. Data from a recently demonstrated $\mathrm{SiC}$ sensor has shown successful temperature measurements from room temperature to $1000^{\circ} \mathrm{C}$, with pressures from 1 to $50 \mathrm{~atm}$. SiC chip refractive index and thickness measurements up to $1000^{\circ} \mathrm{C}$ have also been possible with nanometer-equivalent sensitivities. ${ }^{36}$

This paper introduces a novel wireless optical sensor design for measuring pressure within extreme environments. ${ }^{37}$ Pressure sensing is accomplished by monitoring the spatial optical properties of a free-space laser beam retroreflected from the $\mathrm{SiC}$ optical chip placed in a sealed capsule interconnected with the environment undergoing pressure changes. Specifically, environmental conditions imposed on the chip cause changes in the chip's optical and mechanical properties. By detecting and processing these changes over the entire illuminated chip region, the given extreme environmental pressure conditions can be deduced. A detailed prior-art citation for pressure sensors is given to put the proposed innovation in perspective. It is highlighted that these prior-art electronic and optical sensor designs require extreme environmental protection packaging of the sensing element to prevent sensing

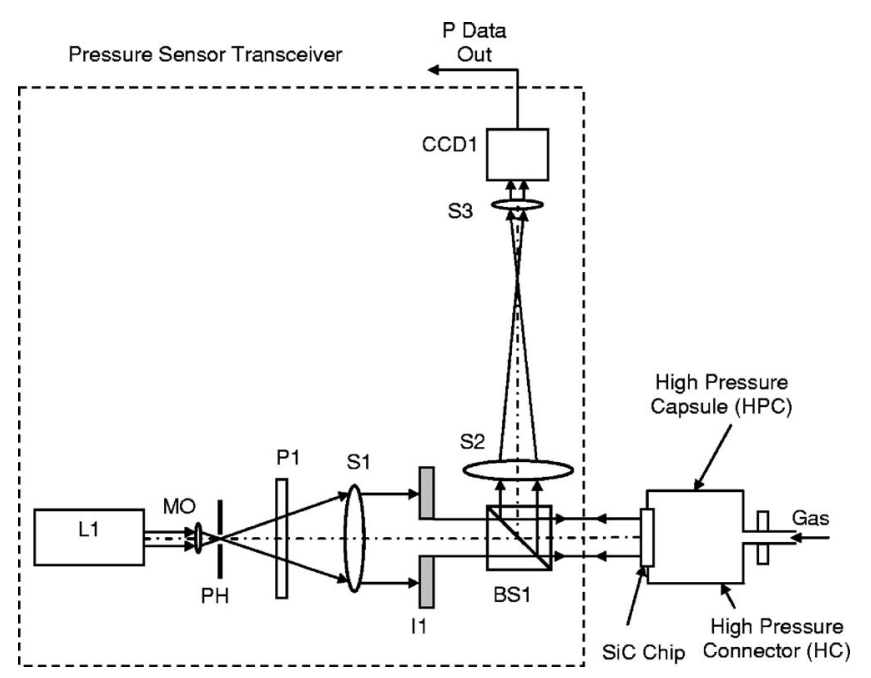

Fig. 1 Proposed SiC-chip-based wireless optical pressure sensor.

element damage, thus making a very challenging sensor design. In addition, the sensor now becomes an indirect reader of the extreme environment conditions, as it is not in direct contact with the conditions. In the proposed sensor, the $\mathrm{SiC}$ chip solves these prior-art problems as the $\mathrm{SiC}$ material itself is the direct sensing element as well as the packaging window within a basic high-pressure capsule such as one made from stainless steel or a suitable hightemperature, high-pressure ceramic. Preliminary experiments from the proposed pressure sensor are presented, including demonstrated pressure measurement ranges and sensitivity, and comparison with theoretical models for chip mechanical deformation. Issues related to sensor packaging and controls are highlighted.

\section{Proposed Wireless Pressure Sensor Design}

Figure 1 shows the proposed wireless pressure sensor concept that uses a remotely placed all-passive optical sensor capsule made of a single-crystal $\mathrm{SiC}$ chip acting as the capsule window and a pressure-sealed capsule assembly made of a suitable high-pressure, high-temperature material such as a ceramic (including $\mathrm{SiC}$ forms, e.g., sintered $\mathrm{SiC}$ ) or high-pressure stainless steel. The capsule has a highpressure connector that interfaces to a high-pressure hot gas (or fluid) flow system that is linked to the high-temperature, high-pressure hot gas flow system such as a fossil fuel plant under test. The $\mathrm{SiC}$ optical window sits in a specially designed, sealed pressure seat that creates the desired highpressure boundary conditions for the deployed SiC chip.

The sensor in Fig. 1 operates as follows. The input laser beam from a laser L1 is passed through an expansion-filter system consisting of a microscope objective lens MO and pinhole PH. The cleaned and expanded beam is vertically polarized using a polarizer P1 and then collimated using a biconvex lens S1. The portion of the light beam that transmits through the beamsplitter BS1 hits the SiC chip seated in the high-pressure capsule with a sealed circular boundary. The size of the beam hitting the $\mathrm{SiC}$ chip is controlled by an iris I1 that is placed between S1 and BS1. Under ambient atmospheric pressure conditions (atmospheric pressure, or $1 \mathrm{~atm}$ ), the reflections from the front and back 
surfaces of the $\mathrm{SiC}$ chip give a phase map that represents the relative optical path length (OPL) differences between the two surfaces. This phase information is seen on a 2-D CCD detector CCD1 in the form of fringes. The initial fringe pattern can be written as $I_{i}(x, y)$, the initial phase map of the given $\mathrm{SiC}$ chip. For a perfect flatness parallel faces chip, one would not observe any fringes, just a grayscale uniform optical power level. Imaging lenses S2 and $\mathrm{S} 3$ are used to form a 1:1 imaging system between the $\mathrm{SiC}$ chip and the CCD. For the laboratory experiments discussed later, a compressed air cylinder is connected to the pressure capsule via a manual regulator to control the pressure inside the capsule relative to the external ambient atmospheric pressure.

The key principle of operation of the proposed sensor is the global sensing of the chip deformation due to pressure. As the pressure in the capsule increases with respect to the external ambient pressure, the $\mathrm{SiC}$ chip undergoes a mechanical deformation and assumes a convex mirror position for the striking incident collimating beam. In effect, the $\mathrm{SiC}$ convex mirror acts as a diverging refractive weak lens that produces a beam expansion for the reflected incident beam. Hence, one should expect a magnification of the beam received at the remote CCD. However, given that the Fig. 1 design uses a 1:1 imaging system between the chip and the CCD, the chips convex-mirror-like deformation combined with the inverting imaging system produces a reduction in the beam size at the CCD with increasing pressure. Hence by monitoring the optical beam's image size on the $\mathrm{CCD}$, a pressure measurement can be achieved remotely. Because CCDs are highly light-sensitive devices and single-crystal $\mathrm{SiC}$ is sufficiently reflective (e.g., $>10 \%$ reflectivity) for visible laser wavelengths, only a low-power (e.g., $<10 \mathrm{~mW}$ ) laser is required for the proposed sensor design. Note that for highly remote distance operations (when the transceiver and capsule distances exceed, for example, $1 \mathrm{~m}$ ), lenses S2 and S3 can be removed. In this case, the received beam expansion is monitored to access the pressure. For the present paper, the basic design in Fig. 1 is investigated as appropriate for short-distance remoting as in a controlled laboratory environment.

\section{Wireless Pressure Sensor Mechanical and Optical Response}

The $\mathrm{SiC}$ chip undergoes a mechanical deformation as the pressure in the capsule is increased. In order to evaluate the nature and extent of this deformation, a theoretical analysis of the mechanical response of the SiC chip within the pressure capsule becomes essential. The nature and extent of this mechanical response is in turn responsible for the optical response of the sensor system. Hence, for optimal pressure sensor design a mathematical relationship between the mechanical and optical responses within the sensor system needs to be established.

The mechanical response of the deployed $6 \mathrm{H} \mathrm{SiC} \mathrm{chip} \mathrm{is}$ dependent on its mounting in the given test pressure capsule. Specifically, the mounting method defines the boundary condition necessary for solving the equations that give the amount of deflection of the $\mathrm{SiC}$ chip, which in turn determines the power of the pressure-sensitive convex mirror behavior of the chip. Considering the design of the chip seat, two major methods, namely, a circular chip with
Applied Uniform Differential Pressure P

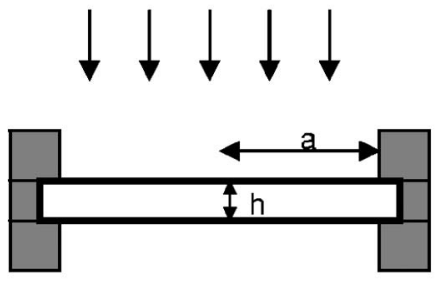

(a)

Applied Uniform Differential Pressure P

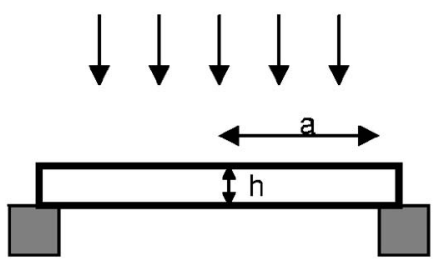

(b)

Fig. 2 The two key mechanical models, (a) clamped edge and (b) simply supported model, used to analyze the SiC chip mechanical deformation when seated in the proposed high-pressure capsule.

clamped edges and a circular chip with simply supported edges, are analyzed for the test capsule [see Fig. 2(a) and 2(b)]. In effect, the $\mathrm{SiC}$ chip experimental mounting method, shown later for the test capsule in this study, results in a hybrid solution of the mentioned methods.

Furthermore, the deflection analysis is subdivided under two regimes of small- and large-deflection analysis. The small-deflection regime is defined by the condition that the maximum deflection should be less than half the thickness of the chip. ${ }^{38}$ Specifically, the region in the middle plane of the sensor chip undergoes small displacements perpendicular to the direction of the plane, thus forming the middle surface of the chip. When these displacements are small in comparison with the thickness of the chip, the strain of the middle plane can be neglected and analysis is in the smalldeflection regime. When this is not true, the analysis is extended to include the effect of strain of the middle plane of the chip. This large-deflection regime analysis gives deflection and stress results that deviate from the smalldeflection regime. As shown later via the experiments, the proposed pressure sensor operates well within the smalldeflection regime of the utilized $6 \mathrm{H} \mathrm{SiC}$ chip.

Also note that failure stress analysis of the $\mathrm{SiC}$ chip is essential for a robust sensor design. Hence, the maximum stress values generated for all pressure cases have to be evaluated. Proper design requires working with pressures that generate maximum stress values that are less than the failure yield stress value for $6 \mathrm{H} \mathrm{SiC}$. This pressure-limited operation ensures the reliable and repeatable performance of the $\mathrm{SiC}$ chip and hence the proposed wireless optical pressure sensor.

Under uniformly distributed applied pressure, a circular 
sensor chip with clamped edges [see Fig. 2(a)] exhibits deflection according to the following classical expression: ${ }^{38}$

$w(r)=\frac{P\left(a^{2}-r^{2}\right)^{2}}{64 D}$,

where $w(r)$ is the out of plane deflection in the chip at a certain radius distance $r, P$ is the applied differential pressure between the two isolated sides of the chip, $a$ is the radius of the chip, and $D$ is its rigidity constant, defined as

$D=\frac{E h^{3}}{12\left(1-\nu^{2}\right)}$,

where $E$ is the chip material's modulus of elasticity, $h$ is its thickness, and $\nu$ is its Poisson's ratio. The maximum deflection is at the center of the chip and is given by

$w_{\max }=\frac{P a^{4}}{64 D}$.

The maximum stress caused by pressure is at the boundary of the chip and is given by the equation

$\left(\sigma_{r}\right)_{\max }=\frac{3 P a^{2}}{4 h^{2}}$.

For a circular sensor chip with supported edges [see Fig. 2(b)], the deflection under uniformly distributed applied pressure is given by the following expression ${ }^{38}$ :

$w(r)=\frac{P\left(a^{2}-r^{2}\right)}{64 D}\left(\frac{5+\nu}{1+\nu} a^{2}-r^{2}\right)$.

The maximum deflection is at the center of the chip and is given by

$w_{\max }=\frac{P a^{4}}{64 D}\left(\frac{5+\nu}{1+\nu}\right)$.

The maximum stress in the supported chip caused by pressure is at the center of the chip and is given by the equation

$\left(\sigma_{r}\right)_{\max }=\frac{3 P a^{2}(3+\nu)}{8 h^{2}}$.

Now consider the proposed case of a $6 \mathrm{H} \mathrm{SiC}$ chip with thickness $h$ of $280 \mu \mathrm{m}$, radius $a$ of $2.5 \mathrm{~mm}$, Poisson's ratio $\nu$ of 0.16 , and Young's modulus $E$ of $415 \mathrm{GPa}^{39,40}$ Figure 3 shows the expected deflection produced for $6 \mathrm{H} \mathrm{SiC}$ sensor chip with the applied differential pressure in the pressurized capsule. The maximum deflection of the sensor chip, with a 5-mm-diameter pressure boundary and thickness of $280 \mu \mathrm{m}$, is expected to be well within the small-deflection range (i.e., <thickness $/ 2=140 \mu \mathrm{m}$ ) at $100 \mathrm{~atm}$. The expected stress produced in the $6 \mathrm{H} \mathrm{SiC}$ sensor chip with the applied differential pressure in the pressurized capsule is shown in Fig. 4. Using a 1-GPa yield stress ${ }^{41}$ (approximate) for the $6 \mathrm{H} \mathrm{SiC}$ chip, the demonstrated wireless pressure sensor is expected to work safely up to a pressure of $100 \mathrm{~atm}$.

Comparing the two $\mathrm{SiC}$ chip seating setups of Fig. 2 in the small-deflection regime, the supported-edge chip seat-

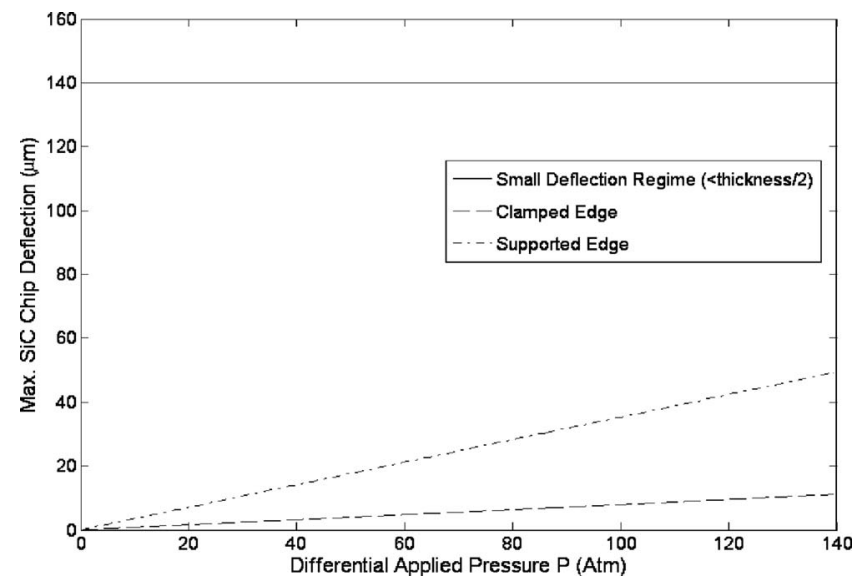

Fig. 3 Maximum SiC chip deflection $w_{\max }$ (at the center of the chip) versus applied pressure for the clamped-edge and supported-edge boundary condition models. The chip boundary diameter was taken to be $5 \mathrm{~mm}$.

ing case gives approximately 3.7 to 5 times larger deflection than the clamped chip case (see Fig. 3) but then it also gives 1.5 to 1.75 times higher maximum stress value (see Fig. 4). Since the experimental setup to seat the $\mathrm{SiC}$ chip in the high-pressure cell utilized in the present study is a hybrid of the two cases, the maximum stress value is expected to be in a range whose limits are defined by the stress values given by the mentioned two cases. The same discussion holds true for the maximum deflection of the SiC chip. Note that in the large-deflection regime, exact analytical solutions are not available and only approximate analytical solutions can be utilized. However, numerical methods and simulation tools (like finite element method software) can provide more exact solutions for the plate/chip deflections and stress values.

After deflection according to Eq. (1) or (5) in the smalldeflection regime, the surface of the $\mathrm{SiC}$ chip and hence the optical response of the $\mathrm{SiC}$ chip can be approximated by a weak lens. Geometrical analysis for weak lensing mirror optics (i.e., when the lensing mirror radius of curvature $R$ is

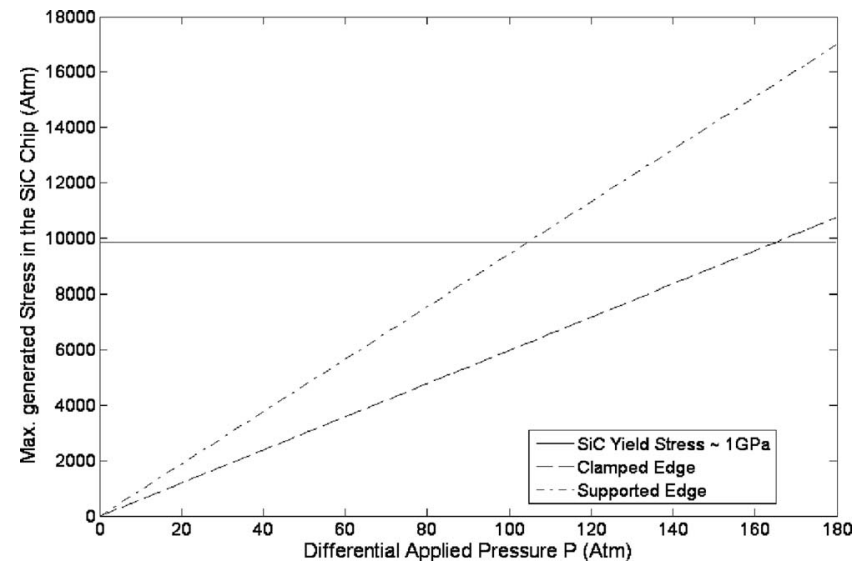

Fig. 4 The expected stress produced in a SiC sensor chip of 5-mm diameter and $280-\mu \mathrm{m}$ thickness. 
Table 1 Theoretical central deflections and focal lengths of the SiC chip convex mirror versus pressure.

\begin{tabular}{|c|c|c|c|c|c|}
\hline \multirow[b]{3}{*}{ (psi) } & \multirow{3}{*}{ (atm) } & \multicolumn{2}{|c|}{ Model 1: } & \multicolumn{2}{|c|}{ Model 2: } \\
\hline & & \multicolumn{2}{|c|}{ Supported sensor chip } & \multicolumn{2}{|c|}{ Clamped sensor chip } \\
\hline & & $\begin{array}{c}\text { Central } \\
\text { deflection } \\
w_{\max }(\mu \mathrm{m})\end{array}$ & $\begin{array}{l}\text { Focal } \\
\text { length } \\
f_{m}(\mathrm{~cm})\end{array}$ & $\begin{array}{c}\text { Central } \\
\text { deflection } \\
w_{\max }(\mu \mathrm{m})\end{array}$ & $\begin{array}{c}\text { Focal } \\
\text { length } \\
f_{m}(\mathrm{~cm})\end{array}$ \\
\hline 0 & 0 & 0.000 & $\infty$ & 0.000 & $\infty$ \\
\hline 200 & 13.61 & 4.804 & 32.53 & 1.080 & 144.68 \\
\hline 300 & 20.41 & 7.206 & 21.68 & 1.620 & 96.45 \\
\hline 400 & 27.21 & 9.608 & 16.26 & 2.160 & 72.34 \\
\hline 500 & 34.01 & 12.010 & 13.01 & 2.699 & 57.87 \\
\hline 600 & 40.82 & 14.412 & 10.84 & 3.240 & 48.23 \\
\hline
\end{tabular}

much less than the lensing mirror central thickness $\left.w_{\max }\right)$ can be carried out to show that the focal length $f_{m}$ of the $\mathrm{SiC}$ weak convex mirror is given by

$f_{m}=\frac{R}{2}=\frac{w_{\text {max }}^{2}+a^{2}}{4 w_{\text {max }}^{2} \times 10^{4}} \mathrm{~cm}$,

where $w_{\max }$ is the $\mathrm{SiC}$ chip central position maximum displacement with applied differential pressure $P$, and $a$ is the radius in microns of the $\mathrm{SiC}$ chip pressure boundary. Using Eqs. (3), (6), and (8) and a pressure boundary of $a$ $=2.5 \mathrm{~mm}$, Table 1 gives the theoretically expected maximum chip central deflection and equivalent weak focal length values for the $\mathrm{SiC}$ chip under specific varying pressure values. These pressure values were implemented later in the experiment. In addition, Fig. 5 shows the theorypredicted focal length change for the $\mathrm{SiC}$ weak mirror for a broad range of pressures.

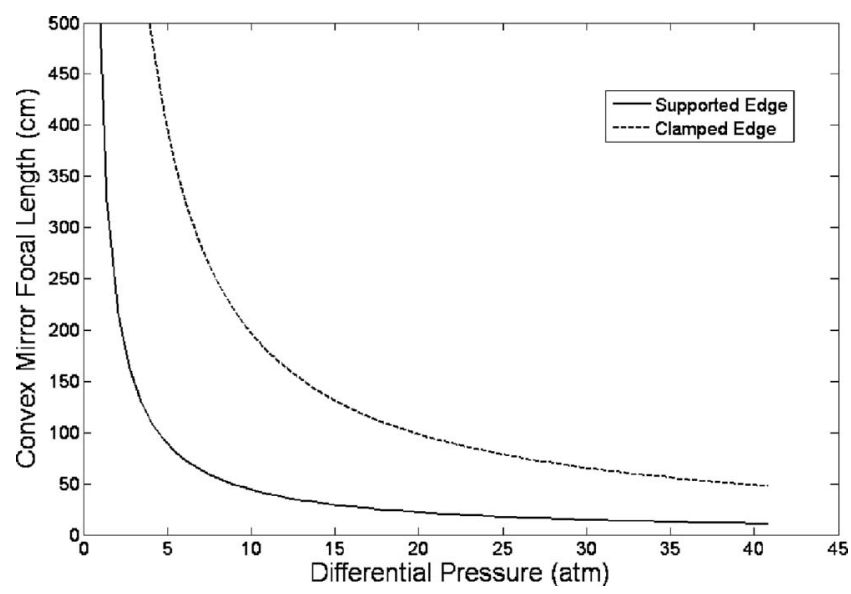

Fig. 5 Effective theoretical focal length $f_{m}$ of the SiC chip acting as a convex mirror due to applied pressure. The focal length decreases as the differential pressure is increased.
Recall that the $\mathrm{SiC}$ chip acts as a weak convex mirror (equivalently, a weak concave lens) within the proposed (Fig. 1) wireless optical sensor setup containing the S2/S3 1:1 imaging system. The lenses $\mathrm{S} 2$ and $\mathrm{S} 3$ have focal lengths $F_{2}$ and $F_{3}$, respectively $\left(F_{2}=F_{3}=F\right)$. When the SiC chip experiences no differential pressure $(P=0)$, it acts like a flat mirror and the S3/S4 lenses form a $1: 1$ imaging system with magnification $M=1$. As $P$ increases, the $\mathrm{SiC}$ chip starts acting like a convex mirror (or concave lens) with a long negative value focal length. It is well known that the equivalent focal length $f_{e}$ for two lenses (convex lens focal length $F_{2}$ and mirror focal length $f_{m}$ ) placed $L$ apart is given by ${ }^{42}$

$f_{e}=\frac{F_{2}\left(L-f_{m}\right)}{L-\left(F_{2}+f_{m}\right)}$.

Hence, the $\mathrm{SiC}$ chip weak concave lens in combination with the first imaging lens $\mathrm{S} 2$ forms an equivalent imaging lens with a new focal length $f_{e}$. With $F_{2}=F$ and $L=F$, and considering weak lensing conditions, which are true for the proposed $\mathrm{SiC}$ pressure sensor, the new pressure-dependent optical linear demagnification $M\left(f_{m}\right.$ is a negative value) for the imaging system can be approximately written as

$M=\frac{F_{3}}{f_{e}}=\frac{F}{f_{e}}=\frac{f_{m}}{f_{m}-F}=\frac{1}{1-F / f_{m}}$.

As $M$ can be measured by computer-based image processing of the CCD-acquired images in Fig. 1 and $F$ is known, $f_{m}$ can be calculated. Furthermore, as $f_{m}$ is related to $w_{\max }$ of the $\mathrm{SiC}$ chip [see Eq. (8)] and $w_{\max }$ is related to the differential pressure $P$ in the capsule [see Eqs. (3) and (6)], the measured pressure $P$ can be calculated. As actual experimental conditions for the $\mathrm{SiC}$ chip seating can be a combination of the clamped and supported chip deformation models, each proposed wireless sensor should be calibrated using a state-of-the-art pressure gauge. In effect, a sensor calibration table should be deployed that stores pre- 


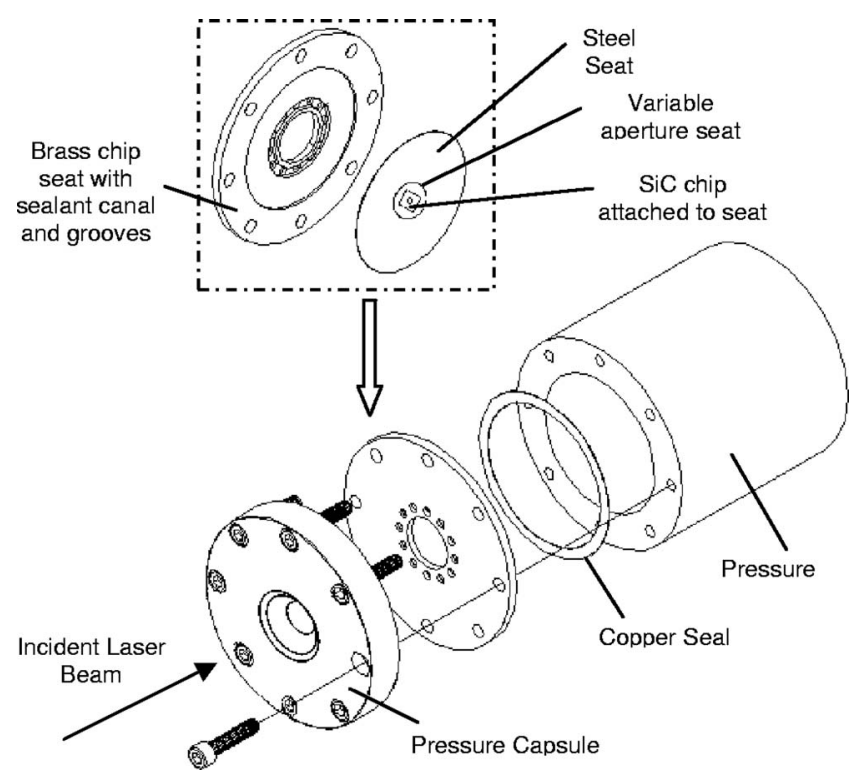

Fig. 6 Experimental design used for seating the SiC chip in the high-pressure capsule.

cision taken pressure values and their corresponding $M$ values provided by the wireless optical pressure sensor. In this way, any nonlinear effects within the pressure versus $M$ function can be calibrated into the sensor measurements, leading to reliable and accurate pressure measurements.

\section{Wireless Pressure Sensor Demonstration}

In order to study the pressure measurement aspects of the proposed wireless sensor in Fig. 1, a high-pressure stainless steel capsule is fabricated as shown in Fig. 6. Figure 7 shows the manner in which a square $1 \times 1-\mathrm{cm} \mathrm{SiC}$ chip is seated in the pressure capsule. The variable aperture seat (washer) used has a 5-mm diameter and creates the circular pressure boundary on the chip. The washer is attached to the steel seat by a GE RTV 102 silicone rubber adhesive sealant with operational temperature range of -60 to $204^{\circ} \mathrm{C}$. The $\mathrm{SiC}$ chip is attached to the washer us-

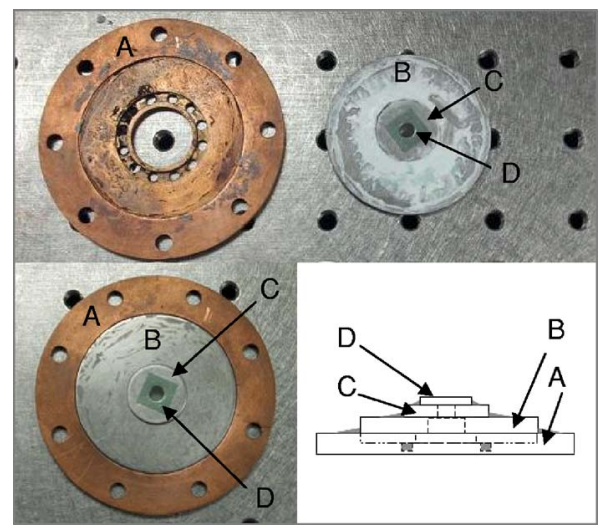

Fig. 7 Snapshot of the experimental seating components and their arrangements used for seating the $6 \mathrm{H} \mathrm{SiC} \mathrm{chip} \mathrm{in} \mathrm{the} \mathrm{high-pressure}$ capsule. Components in photograph are labeled as follows: A, brass chip seat holder with sealant canal and grooves; B, steel seat; C, aperture seat (washer), and D, SiC chip. ing a layer of the same sealant. The sealant is filled in the canal of the brass seat so it strongly holds onto the steel seat. A layer of the sealant was also applied at the edges of the two seats to avoid any leaks. Note that for hightemperature or temperature-independent pressure sensor operation, a modified SiC seating design should be utilized to match the seat and chip material's thermal coefficient of expansion (CTE) values to avoid temperature-dependent global chip deformation. This aspect will be pursued in future advanced-stage work.

To enable the present sensor design of Fig. 1, a 10-mW 633-nm (red wavelength) linear-polarization $\mathrm{He}-\mathrm{Ne}$ laser is used as the source L1. The expansion-filter system utilizes a $10 \times \mathrm{MO}$ lens and a $10-\mu \mathrm{m}$ PH. The collimating lens has a focal length of $15 \mathrm{~cm}$. The sealed circular boundary of the $\mathrm{SiC}$ chip for the experiment is of $\approx 5$-mm diameter. Imaging lenses S2 and S3 have $10-\mathrm{cm}$ focal lengths, forming a 40-cm-path $1: 1$ imaging system between the $\mathrm{SiC}$ chip and the $\mathrm{CCD}$. The temperature condition during the experiment is the ambient $26^{\circ} \mathrm{C}$ room temperature. The manual regulator connected to the compressed air cylinder is used to control the pressure inside the capsule relative to the external ambient 1 -atm pressure.

As shown in Fig. 8, as the pressure in the capsule increases above 1 atm, the differential pressure $P$ on the $\mathrm{SiC}$ chip produces an increasing convex-mirror chip deformation of the initial pattern $I_{i}(x, y)$, thus producing a pattern reduction in size. Figure 8 shows the set of images from the wireless pressure sensor where the initial fringe pattern size decreases with increasing capsule differential pressures up to $40.8 \mathrm{~atm}$ (1 psi=0.068 atm). A quantization of the image reduction versus applied differential pressure $P$ in the capsule for $P$ taken up to $40.8 \mathrm{~atm}$ is shown in Table 2 , giving the experimentally measured values for $M$ versus $P$. Figure 9 connects the discrete experimental data to show a plot of differential pressure $P$ on $\mathrm{SiC}$ chip versus image size given by pixel count. These results show a linear behavior of the applied differential pressure $P$ versus the measured optical parameter of image size. The sensor pressure resolution is given by the inverse of the slope of the plot in Fig. 9. This plot indicates a current experimental resolution of 1.17 atm, calculated as $40.82 \mathrm{~atm} /[(147-112)$ pixels $]$. The resolution measurement is restricted by the pixel size in the deployed CCD. Here 147 pixels of the CCD $=5 \mathrm{~mm}$ real size using the $1: 1$ imaging approach. One can greatly improve the pressure measurement resolution by decreasing the pixel size and increasing the size of the optical beam on the CCD plane. This is to our knowledge the first demonstration of a wireless SiC optical chip for high-pressure assessment and points to the robustness of the thick SiC chip under high gas pressures, a result needed for potential fossil-fuel power generation system gas species detection using custom $\mathrm{SiC}$ chips. For comparison and sensor design accuracy, Fig. 10 shows plots for the experimentally detected image magnification $M$ versus pressure $P$ for the studied wireless sensor versus the theoretical design plots. These plots indicate that the $\mathrm{SiC}$ chip seating is closer to the clamped case, an expected results because of the pressure washer (seal) used to maintain the chip in a sealed arrangement. In addition, the results in Fig. 10 indicates linear operation of the demonstrated pressure sensor, with 


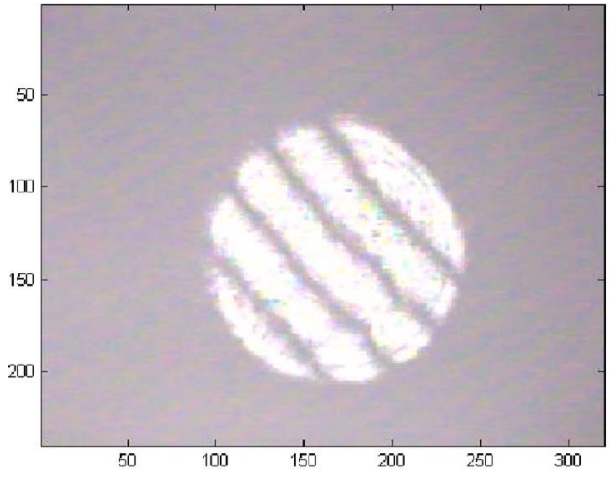

(a)

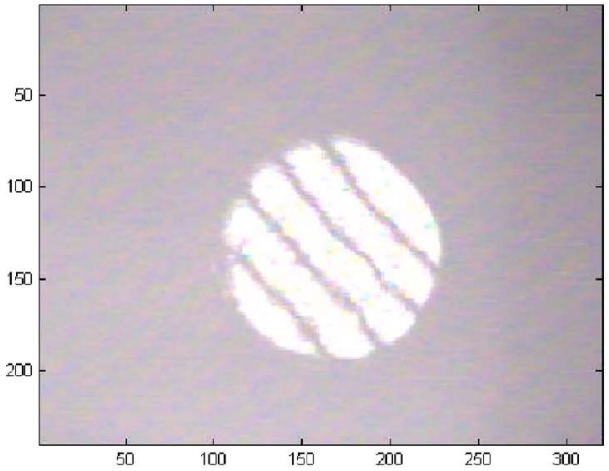

(c)

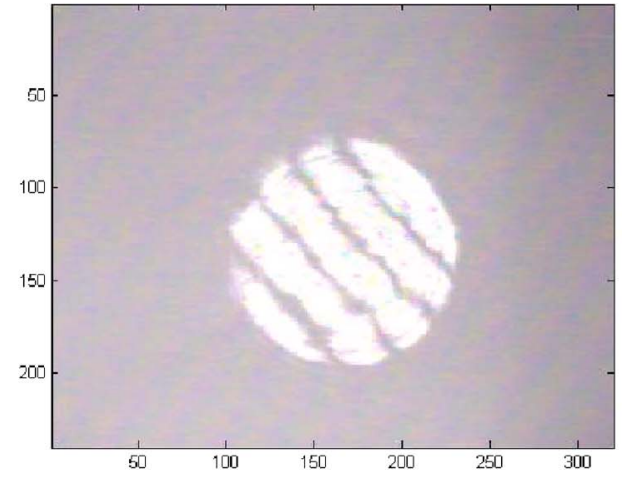

(b)

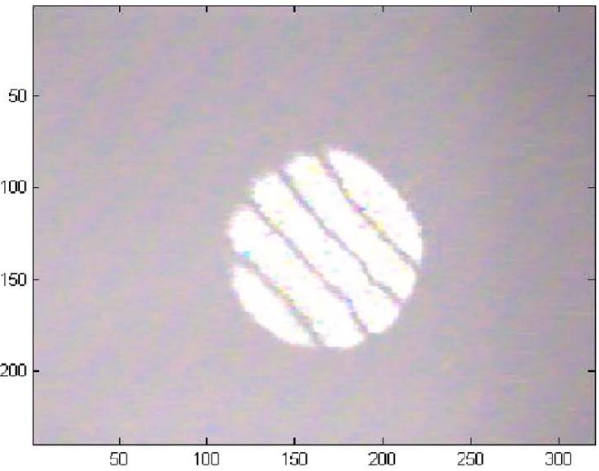

(d)

Fig. 8 Optical images $l_{i}(x, y)$ produced by the SiC-chip-based pressure system for (a) 0 atm, (b) $13.6 \mathrm{~atm}$ (200 psi), (c) $27.2 \mathrm{~atm}$ (400 psi), and (d) $40.8 \mathrm{~atm}$ (600 psi) high differential pressure conditions in the pressure capsule. Dimensions on both axes are in CCD pixels.

the $\mathrm{SiC}$ chip performance well within the linear elastic small-deformation range, a need for long-life repeatable robust sensor operations.

\section{Conclusion}

An extreme-environment wireless pressure sensor design has been proposed, using a thick single-crystal $6 \mathrm{H} \mathrm{SiC} \mathrm{chip}$

Table 2 Experimental optical image size versus capsule differential pressure $P$.

\begin{tabular}{cccc}
\hline \hline $\begin{array}{c}\text { Differential pressure } P \\
(\text { psi })\end{array}$ & Image size & $\begin{array}{r}\text { Measured magnification } \\
\text { (atm) }\end{array}$ & $\begin{array}{c}\text { (CCD pixels }) \\
M=\text { image size/147 }\end{array}$ \\
\hline 0 & 0 & 147 & 1.00 \\
200 & 13.61 & 135 & 0.92 \\
300 & 20.41 & 130 & 0.88 \\
400 & 27.21 & 125 & 0.85 \\
500 & 34.01 & 117 & 0.80 \\
600 & 40.82 & 112 & 0.76 \\
\hline \hline
\end{tabular}

seated in a pressure capsule that can be designed for extreme conditions. The sensor operates on the principle of remote laser beam targeting, $\mathrm{SiC}$ chip optical weak lensing, and received-beam image measurement. The concept has been tested up to $41 \mathrm{~atm}$ with an initial 1.17 -atm pressure measurement resolution. Experimental results also show a

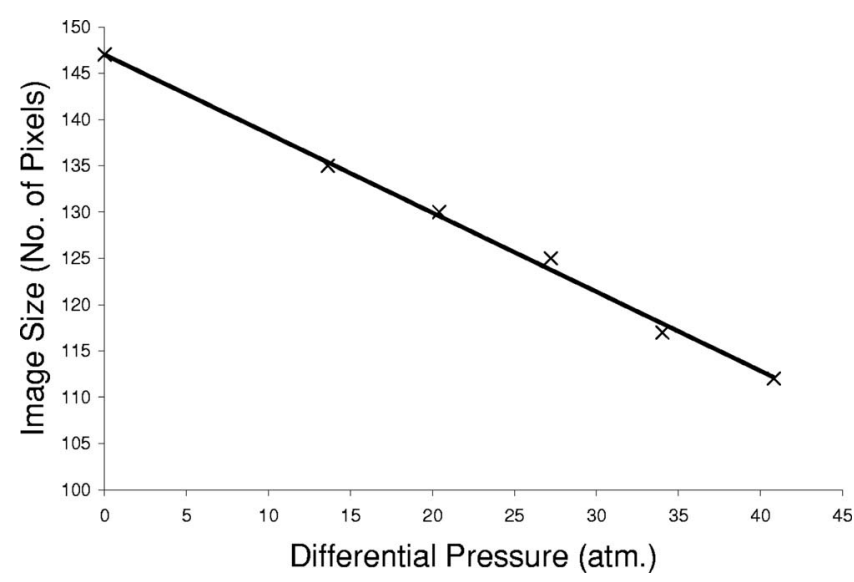

Fig. 9 Plot of demagnification of incident beam as it reflects from the $\mathrm{SiC}$ chip under pressure acting as a weak convex mirror coupled to a 1:1 image inversion system. 


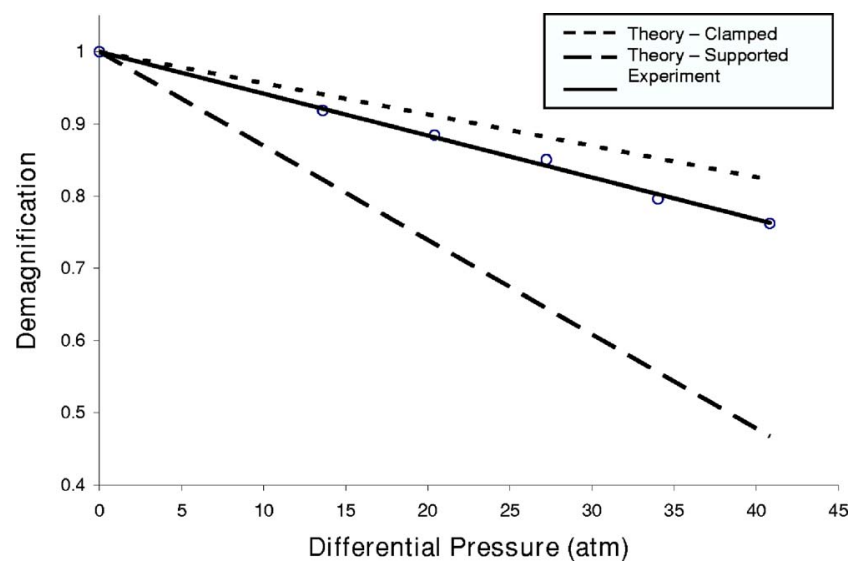

Fig. 10 Experimental beam demagnification along with the theoretical demagnification for clamped and supported sensor chip models. The behavior of the chip shifts from the clamped model towards the supported model as the pressure increases.

linear mechanical and optical behavior of the $\mathrm{SiC}$ chip and sensor demagnification parameter readout, indicating the expected robustness of the $\mathrm{SiC}$ chip technology under high pressures. Mechanical deflection analysis points to a clamped seating case for the $\mathrm{SiC}$ chip within the capsule, while chip stress analysis indicates a robust design reaching $140 \mathrm{~atm}$ and beyond. The sensor resolution can be improved with optimized $\mathrm{SiC}$ chip and capsule designs. These initial results attest to the promise and strengths of SiCchip-based optical wireless sensor technology for fossilfuel-based power generation system applications. Future work relates to designing a temperature-independent wireless pressure sensor with optimal capsule packaging and extensions to long-distance remote sensor operations.

\section{Acknowledgments}

This paper was prepared with the support of the U.S. Department of Energy under Award No. DE-FC3603NT41923. However, any opinions, findings, conclusions, or recommendations expressed herein are those of the authors and do not necessarily reflect the views of the DOE. Support for the high-pressure test capsule was provided by AppliCote Associates (Dr. N. Quick). N. A. Riza is on partial leave from UCF.

\section{References}

1. B. Culshaw, "Fiber-optic sensors: applications and advances," OSA Opt. Photon. News 16, pp. 24-29 (Nov. 2005).

2. P. Fairley, "The greening of GE," IEEE Spectrum 42(7), pp. 28-33 (July 2005)

3. J. H. Ausubel, "Big green energy machines," Ind. Phys. 10(5), pp. 20-24, (Oct.-Nov. 2004)

4. R. S. Okojie, A. A. Ned, and A. D. Kurtz, "Operation of $\alpha(6 \mathrm{H})-\mathrm{SiC}$ pressure sensor at $500^{\circ} \mathrm{C}$," in IEEE int. Con. on SolidState Sensors and Actuators, pp. 1407-1409 (1997).

5. R. S. Okojie, A. A. Ned and A. D. Kurtz, "Operation of $\alpha(6 \mathrm{H})-\mathrm{SiC}$ pressure sensor at $500^{\circ} \mathrm{C}$," Sens. Actuators, A 66(1-3), 200-204 (1998).

6. R. Ziermann, J. von Berg, W. Reichert, E. Obermeier, M. Eickhoff, and G. Krötz, "A high temperature pressure sensor with $\beta$-SiC piezoresistors on SOI substrates," in IEEE Int. Con. on Solid-State Sensors and Actuators, pp. 1411-1414 (1997).

7. W. Pulliam, P. Russler, R. Mlcak, K. Murphy, and C. Kozikowski, "Micromachined SiC fiber-optic pressure sensors for high temperature aerospace applications," Proc. SPIE 4202, 21-30 (Dec. 2000).
8. A. Dehennis and K. D. Wise, "A double-sided single-chip wireless pressure sensor," in 15th IEEE Int. Con. on MEMS, pp. 252-255. (2002).

9. O. Akar, T. Akin, and K. Najafi, "A wireless batch sealed absolute capacitive pressure sensor," in 14th Eur. Con. on Solid State Transducers (EuroSensors), pp. 585-588 (2000).

10. G. Schimetta, F. Dollinger, and R. Weigel, "A wireless pressuremeasurement system using a SAW hybrid sensor," IEEE Trans. Microwave Theory Tech. 48, 2730-2735 (Dec. 2000).

11. M.A. Fonseca, J. M. English, M. von Arx, and M. G. Allen, "Wireless micromachined ceramic pressure sensor for high-temperature applications," J. Microelectromech. Syst. 11, 337-343 (2002).

12. C. E. Lee and H. F. Taylor, "Sensors for smart structures based on the Fabry-Perot interferometer," Chap. 9, Fiber Optic Smart Structures, Eric Udd, Ed. pp. 249-270 Wiley, New York, 1995.

13. R. Duncan, D. Gifford, and V. Rajendran, "OFDR tracks temperatures on power generators," Laser Focus World Mag. 39(1010), 8992, (2003).

14. A. D. Kersey, M. A. Davis, H. J. Patrick, M. LeBlanc, K. P. Koo, C. G. Askins, M. A. Putnam, and E. J. Friebele, "Fiber grating sensors," J. Lightwave Technol. 15(8), 1442-1463 (1997).

15. B. Culshaw, "Optical fiber sensor technologies: opportunities and perhaps pitfalls," J. Lightwave Technol. 22(1), 39-50 (2004).

16. L. Tenerz, L. Smith, and B. Hök, "A fiber optic silicon pressure microsensor for measurements in coronery arteries," in Proc. Sixth Int. Conf. on Solid State Sensors and Actuators, Digest of Technical Papers, Transducers'91, pp. 1021-1023 (1991)

17. R. A. Wolthuis, G. L. Mitchell, E. Saaski, J. C. Harti, and A. Afromowitz, "Development of medical pressure and temperature sensors employing optical spectrum modulation," IEEE Trans. Biomed. Eng. 38, 974-981 (1991)

18. Y. Kim and D. P. Neikirk, "Micromachined Fabry-Perot cavity pressure transducer," IEEE Photonics Technol. Lett. 7(12), 1471-1473 (1995).

19. J. Han, J. Y. Kim, T. S. Kim, and J. S. Kim, "Performance of FabryPerot microcavity structures with corrugated diaphragms," Sens. Actuators, A 79 162-172 (2000).

20. T. Katsumata, Y. Haga, K. Minami, and E. Esashi, "Micromachined $125 \mu \mathrm{m}$ diameter ultra-miniature fiber-optic pressure sensor for catheter," Trans. Inst. Electr. Eng. Jpn., Sect. E 120, 58-63 (2000).

21. J. Zhou, S. Dasgupta, H. Kobayashi, J. M. Wolff, H. E. Jackson, and J. T. Boyd, "Optically interrogated MEMS pressure sensors for propulsion applications," Opt. Eng. 40, 598-604 (2001).

22. D. C. Abeysinghe, S. Dasgupta, J. T. Boyd, and H. E. Jackson, "A novel MEMS pressure sensor fabricated on an optical fiber," IEEE Photonics Technol. Lett. 13, 993-995 (2001).

23. W. Li, D. C. Abeysinghe, and J. T. Boyd, "Wavelength multiplexing of microelectromechanical system pressure and temperature sensors using fiber Bragg gratings and arrayed waveguide gratings," Opt. Eng. 42(2), 431-438 (2003).

24. W. J. Wang, R. M. Lin, D. G. Guo, and T. T. Sun, "Development of a novel Fabry-Perot pressure microsensor," Sens. Actuators, A 116(1), 59-65 (2004).

25. W. Li, D. C. Abeysinghe, and J. T. Boyd, "Multiplexed sensor system for simultaneous measurement of pressure and temperature," Opt. Eng. 43(1), 148-156 (2004)

26. D. Guo, W. Wang, and R. Lin, "Theoretical analysis and measurement of the temperature dependence of a micromachined Fabry-Perot pressure sensor," Appl. Opt. 44(2), 249-256 (2005).

27. Y. Zhu and A. Wang, "Miniature fiber-optic pressure sensor," IEEE Photonics Technol. Lett. 17(2), 447-449 (2005).

28. J. Xu, G. Pickrell, X. Wang, W. Peng, K. Cooper, and A. Wang, "A novel temperature insensitive optical fiber pressure sensor for harsh environments," IEEE Photonics Technol. Lett. 17(4), 870-872 (2005).

29. D. Donlagic and E. Cibula, "All-fiber high sensitivity pressure sensor with $\mathrm{SiO}_{2}$ diaphragm," Opt. Lett. 30(16), 2071-2073 (2005).

30. H. Xiao, J. Deng, Z. Wang, W. Huo, P. Zhang, M. Luo, G. Pickrell, R. May, and A. Wang, "Fiber optic pressure sensor with selfcompensation capability for harsh environment applications," Opt. Eng. 44(5), 054403 (2005).

31. M. Li, M. Wang, and H. Li, "Optical MEMS pressure sensor based on Fabry-Perot interferometry," Opt. Express 14(4), 1497-1504 (2006).

32. Y. Zhu, K. L. Cooper, G. R. Pickrell, and A. Wang, "Hightemperature fiber-tip pressure sensor," J. Lightwave Technol. 24(2), $861-869$ (2006).

33. N. A. Riza, M. A. Arain, and F. Perez, "Harsh environments minimally invasive optical sensing technique for extreme temperatures: $1000^{\circ} \mathrm{C}$ and approaching $2500^{\circ} \mathrm{C}$," Proc. SPIE 5855, 687-690 (2005)

34. N. A. Riza, M. A. Arain, and F. Perez, "Harsh environments minimally invasive optical sensor using freespace targeted single crystal silicon carbide," IEEE Sens. J. 6(3), 672-675 (2006).

35. M. A. Arain and N. A. Riza, "Fiber coupled in-line heterodyne opti- 
Riza, Ghauri, and Perez: Wireless pressure sensor using laser targeting...

cal interferometer for minimally invasive sensing," J. Lightwave Technol. 23(8), 2449-2454 (2005)

36. N. A. Riza, M. Arain, and F. Perez, "6-H single crystal silicon carbide thermo-optic coefficient measurements for ultrahigh temperatures upto $1273 \mathrm{~K}$ in the telecommunications infrared band," J. Appl. Phys. 98(10), 103512 (2005)

37. N. A. Riza, F. N. Ghauri, and F. Perez, "Hybrid optical sensor using laser targeting," in Optical Sensing II, B. Culshaw and A. Mignani, Eds. Proc. SPIE 6189 (2006).

38. S. P. Timoshenko and S. Woinowsky-Krieger, Theory of Plates and Shells, Chap. 2, pp. 56-57, McGraw-Hill, New York (1959).
39. NIST Structural Ceramic Data Base, SRD Data Base No. 30, www.ceramics.nist.gov/srd/summary/scdscs.htm

40. R. G. Munro, "Material properties of a sintered alpha-SiC," J. Phys. Chem. Ref. Data 26, 1195-1203 (1997).

41. W. N. Sharpe, O. Jadaan, G. M. Beheim, G. D. Quinn, and N. N. Nemeth, "Fracture strength of silicon carbide micro-specimens," IEEE J. Microelectromech. Sys. 14(5), 903-913 (2005)

42. E. Hecht, Optics, 2nd ed., Eq. (5.36), p. 148, Addison-Wesley, Reading, MA (1990).

Biographies and photographs of the authors not available. 